\title{
Tubal ligation, hysterectomy and ovarian cancer: A meta-analysis
}

\author{
Megan S Rice ${ }^{1,2,3^{*}}$, Megan A Murphy ${ }^{1,2,3}$ and Shelley S Tworoger ${ }^{1,2,3}$
}

\begin{abstract}
Purpose: The purpose of this meta-analysis was to determine the strength of the association between gynecologic surgeries, tubal ligation and hysterectomy, and ovarian cancer.

Methods: We searched the PubMed, Web of Science, and Embase databases for all English-language articles dated between 1969 through March 2011 using the keywords "ovarian cancer" and "tubal ligation" or "tubal sterilization" or "hysterectomy." We identified 30 studies on tubal ligation and 24 studies on hysterectomy that provided relative risks for ovarian cancer and a p-value or $95 \%$ confidence interval (Cl) to include in the meta-analysis. Summary RRs and $95 \%$ Cls were calculated using a random-effects model.

Results: The summary RR for women with vs. without tubal ligation was 0.70 (95\%Cl: 0.64, 0.75). Similarly, the summary RR for women with vs. without hysterectomy was 0.74 (95\%Cl: $0.65,0.84)$. Simple hysterectomy and hysterectomy with unilateral oophorectomy were associated with a similar decrease in risk (summery $R R=0.62,95 \%$ Cl: $0.49-0.79$ and $0.60,95 \% \mathrm{Cl}: 0.47-0.78$, respectively). In secondary analyses, the association between tubal ligation and ovarian cancer risk was stronger for endometrioid tumors (summary $\mathrm{RR}=0.45,95 \% \mathrm{Cl}$ : $0.33,0.61$ ) compared to serous tumors.

Conclusion: Observational epidemiologic evidence strongly supports that tubal ligation and hysterectomy are associated with a decrease in the risk of ovarian cancer, by approximately $26-30 \%$. Additional research is needed to determine whether the association between tubal ligation and hysterectomy on ovarian cancer risk differs by individual, surgical, and tumor characteristics.
\end{abstract}

Keywords: Ovarian neoplasms, Sterilization, Tubal, Hysterectomy

\section{Introduction}

Ovarian cancer is the fifth leading cause of cancer death in US women [1], yet primary prevention recommendations are limited. Gynecological surgeries including tubal ligation and hysterectomy may alter ovarian cancer risk by protecting the ovary from ascending carcinogens or damaging the utero-ovarian artery altering hormonal function. In addition, tubal ligation may increase immunity against the surface glycoprotein human mucin 1 (MUC1) [2-4]. While tubal ligation and hysterectomy generally have been found to be inversely associated with ovarian cancer, effect estimates vary between studies and

\footnotetext{
*Correspondence: nhmsr@channing.harvard.edu

'Channing Laboratory, Department of Medicine, Brigham and Women's

Hospital and Harvard Medical School, Boston, MA, USA

2Department of Epidemiology, Harvard School of Public Health, Boston, MA, USA

Full list of author information is available at the end of the article
}

little is known about potential effect modifiers of these associations. Therefore, we conducted a meta-analysis of the association between ovarian cancer and tubal ligation as well as hysterectomy.

\section{Materials and methods}

Through searches in the PubMed, Web of Science, and Embase databases, we sought to identify all Englishlanguage articles with quantitative data on the association between tubal ligation or hysterectomy and the risk of ovarian cancer. Database searches encompassed articles dated 1969 through March 2011. We identified articles using the keywords "ovarian cancer" and "tubal ligation" or "tubal sterilization" as well as "ovarian cancer" and "hysterectomy." In addition, we reviewed the references of selected articles to identify studies missed through our search. We also completed a reverse

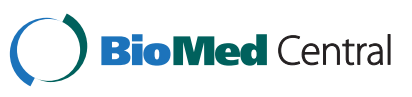


citation query to include pertinent articles, which referenced those already identified, using the Cited Reference Search application available through the Web of Science. All articles selected for inclusion in our analyses were verified by a second reviewer.

We abstracted relative risks (RRs) and 95\% CIs or p-values from selected articles. We used estimates adjusted for multiple confounders when available and calculated standard errors from the $95 \%$ CIs or p-values. We decided apriori to use a random-effects model to calculate the summary $R R$ estimates and 95\% CIs [5]. Q tests for heterogeneity were used to evaluate the consistency of findings among studies and Begg's and Egger's tests were used to assess publication bias [6,7]. We conducted meta-regression analyses to assess whether effect estimates differed by study design (i.e., case-control versus cohort versus other design) and by population studied (i.e., general population versus BRCA mutation carriers) [8]. In secondary analyses, we conducted meta-regression analyses in subsets of the studies to assess whether the effect estimates differed by age at procedure, years since procedure, and, for the tubal ligation analysis, by histological subtype (i.e., serous, mucinous, endometrioid, clear cell, other). All analyses were conducted using the Stata/SE 10.0 for Windows.

\section{Results}

\section{Database search}

We identified 30 studies that provided estimates of the risk of ovarian cancer in relation to tubal ligation as well as the p-value or 95\% confidence interval (CI) [9-37] to include in the meta-analysis (Figure 1). One of the studies examined the risk of ovarian cancer death [28] and three studies were conducted in BRCA carriers [13,18,20]. Therefore, we conducted sensitivity analyses examining the influence of these studies, which are detailed below. For the examination of hysterectomy and ovarian cancer, we identified 24 studies to include in the meta-analysis (Figure 1) [9,10,12,13,15,16,23-26,29,31,32,38-47]. Nine of the studies reported effect estimates for simple hysterectomy, [23,25,29,32,38,42,43,45] seven provided estimates for hysterectomy with unilateral oophorectomy, $[23,29,32,38,42,45]$ and 15 did not distinguish whether or not women with hysterectomy underwent a unilateral oophorectomy $[9,10,12,13,15,16,24,26,31,39-41,44,46,47]$. Two of the studies included in the primary meta-analysis for both tubal ligation and hysterectomy were pooled analyses $[9,31]$, one was comprised of eight studies [31] and another was comprised of four studies [9]. For these studies, we included the pooled estimates in our meta-analysis as we were unable to obtain the study-specific effect estimates for all studies through our literature search. One of the studies identified in our tubal ligation and hysterectomy literature searches was a study in the New England case-control study (NECC) [Cramer]. However, in this study the reference category for the odds ratios for tubal ligation and hysterectomy was comprised of women who did not have any pelvic surgeries, including cesarean sections. In order for the effect estimates from the NECC to be comparable to other studies, we requested and obtained from NECC researchers the odds ratio for ovarian cancer comparing women who had a tubal ligation to those who did not have the procedure as well as the odds ratio comparing women with hysterectomy to those who did not have a hysterectomy. We also obtained odds ratios for the secondary analyses described below.

In secondary analyses, we identified studies that reported the relative risk of ovarian cancer by characteristics of surgery, such as age at or years since procedure, as well as by histological subtype of ovarian cancer. We identified eight studies that reported stratum-specific estimates of ovarian cancer risk by years since tubal ligation (Additional file 1 : Table S1) $[14,19,25,26,28,29,48]$ and nine studies that reported stratum-specific estimates for age at tubal ligation (Additional file 1: Table S2) [13,14,19,25,27-29,48]. In addition, 13 studies specified effect estimates for invasive ovarian cancer [10,12,15,17-23,31,33] and 11 studies on tubal ligation reported estimates for at least one histological subtype of ovarian cancer (Additional file 1: Table S3) $[9,10,15,16,19,22,24,26,29,49]$. Eight studies on hysterectomy reported stratum-specific estimates of ovarian cancer risk by years since the procedure (Additional file 1: Table S4) $[25,26,29,31,43,45,46]$ and five studies reported stratum-specific estimates for age at hysterectomy (Additional file 1: Table S5) [25,29,31,43]. In addition, nine studies reported effect estimates for invasive ovarian cancer [[10,12,15,23,31,40-42], Cramer].

Separate analyses were performed examining risk of ovarian cancer and characteristics of surgery, including years since and age at procedure. For six of the eight studies reporting stratum-specific estimates for years since tubal ligation, we were able to derive estimates for less than 10 years since tubal ligation and 10 or more years since tubal ligation $[19,25,26,29,48]$. For seven of the nine studies that reported risks by age at tubal ligation, we were able to derive estimates for age less than 35 at tubal ligation and 35 years of age or older $[13,19,27-29,48]$. For seven of the eight studies reporting stratum-specific estimates for years since hysterectomy, we were able to derive estimates for less than 10 years since hysterectomy and 10 or more years since hysterectomy $[22,25,26,31,43,45]$. For the five studies that reported risks by age at hysterectomy, we were able to derive estimates for age less than 40 or 45 at hysterectomy and 40 or 45 years of age or older [25,29,31,43] [NECC]. 


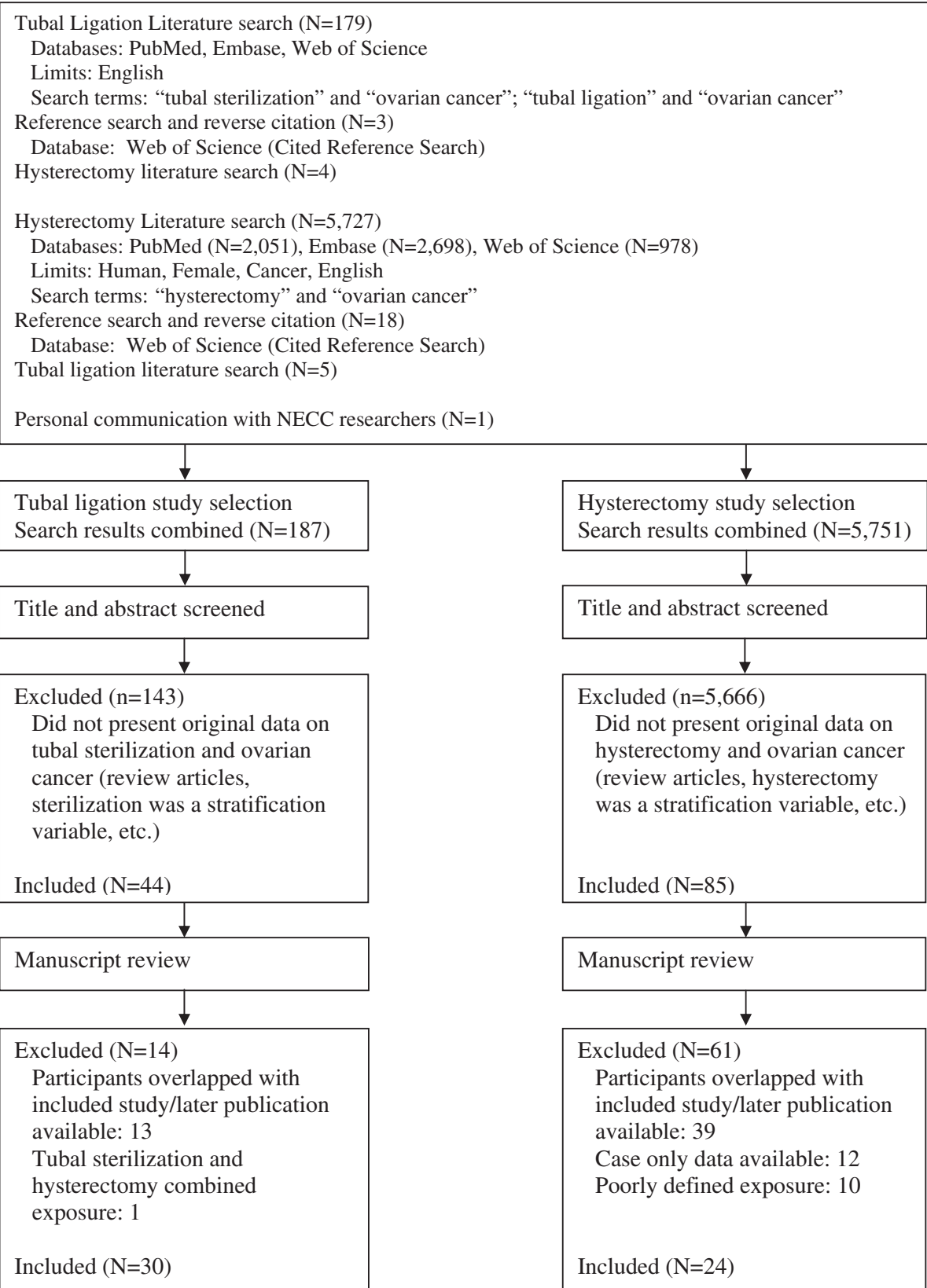

Figure 1 Selection of studies on tubal ligation and hysterectomy with risk of ovarian cancer.

\section{Tubal ligation}

The estimated RRs for ovarian cancer associated with tubal ligation versus no tubal ligation ranged from 0.2 to 2.4 (Table 1). Twenty-seven of the 30 studies reported lower risks of ovarian cancer in women who had a tubal ligation compared to those who had not had the procedure. The three studies that observed an elevated risk of ovarian cancer did not achieve statistical significance $[14,16,35]$. The summary RR was 0.70 (95\%CI: 0.64, $0.75)$, demonstrating a statistically significant inverse association between tubal ligation and ovarian cancer
(Figure 2). Some studies in our analysis did not specify whether borderline cases were included in the analyses. However, when we restricted our analysis to 13 studies that reported the association for invasive ovarian cancer, specifically the summary RR was very similar (summary $\mathrm{RR}=0.72 ; 95 \% \mathrm{CI}: 0.66,0.72)$. Since there was evidence of heterogeneity among the 30 studies $(P=0.02)$, we examined the contribution of study characteristics to the heterogeneity. We did not observe statistically significant evidence of heterogeneity by study design (i.e., cohort study, case-control study, or other) or residence of study 
Table 1 Epidemiologic Studies of the Association Between Tubal Ligation and Risk of Ovarian Cancer

\begin{tabular}{|c|c|c|c|c|c|}
\hline Author (Country) & Study Design & Case definition & Covariates & OR, RR, or SIR $(95 \% \mathrm{Cl})$ & Comments \\
\hline $\begin{array}{l}\text { NECC } 2012 \text { (USA) } \\
\text { [personal } \\
\text { communication } \\
\text { with Dr. Daniel } \\
\text { Cramer] }\end{array}$ & Case-control & $\begin{array}{l}\text { Borderline or invasive } \\
\text { epithelial ovarian cancer } \\
N=2076\end{array}$ & $\begin{array}{l}\text { age, study center, BMl, study phase, smoking, } \\
\text { family history of ovarian and breast cancers, } \\
\text { talc use, OC use, parity, breast feeding, age at } \\
\text { menarche, post-menopausal status, use of } \\
\text { post-menopausal hormones, hysterectomy }\end{array}$ & $0.79(0.66-0.94)$ & \\
\hline \multirow[t]{2}{*}{$\begin{array}{l}\text { Ness et al. } 2011 \\
\text { (USA) [11] }\end{array}$} & \multirow[t]{2}{*}{ Case-control } & $\begin{array}{l}\text { Invasive or borderline } \\
\text { epithelial ovarian cancer }\end{array}$ & $\begin{array}{l}\text { Age, number of pregnancies, race, infertility, } \\
\text { family history of }\end{array}$ & \multirow[t]{2}{*}{$0.63(0.51-0.77)$} & \\
\hline & & $N=867$ & $\begin{array}{l}\text { ovarian cancer, ever use of oral contraceptives, } \\
\text { ever use of IUDs, ever use of barriers, } \\
\text { vasectomy }\end{array}$ & & \\
\hline \multirow{3}{*}{$\begin{array}{l}\text { Moorman et al. } \\
2009 \text { (USA) [12] }\end{array}$} & \multirow{3}{*}{$\begin{array}{l}\text { Case-control North } \\
\text { Carolina Ovarian Cancer } \\
\text { Study }\end{array}$} & Invasive epithelial ovarian & \multirow{3}{*}{$\begin{array}{l}\text { Age, parity, age at menarche, duration of OC } \\
\text { use, family history of breast/ovarian cancer, } \\
\text { BMI }\end{array}$} & \multirow{3}{*}{$\begin{array}{l}\text { Whites: } 0.74(0.58,0.94) \\
\text { African-Americans: } 0.43(0.24 \text {, } \\
0.80)\end{array}$} & \\
\hline & & $\begin{array}{l}\text { cancer } \\
\mathrm{N}=746 \text { White cases }\end{array}$ & & & \\
\hline & & $\begin{array}{l}N=111 \text { African-American } \\
\text { cases }\end{array}$ & & & \\
\hline \multirow{4}{*}{$\begin{array}{l}\text { Antoniou et al. } \\
2009 \text { (Europe and } \\
\text { Canada) [13] }\end{array}$} & \multirow[t]{4}{*}{ Retrospective Cohort } & Ovarian cancer (only BRCA 1/ & \multirow[t]{4}{*}{ Age, duration of $\mathrm{OC}$ use, parity } & BRCA 1/2: $0.43(0.24,0.75)$ & \multirow{4}{*}{$\begin{array}{l}\text { Includes prevalent and incident } \\
\text { cases. } \\
\text { Mean difference between age at } \\
\text { diagnosis and interview: } 6.7 \text { years }\end{array}$} \\
\hline & & 2 carriers) & & BRCA1: $0.42(0.22,0.80)$ & \\
\hline & & $\mathrm{N}=201 \mathrm{BRCA} 1$ cases & & \multirow{2}{*}{ BRCA2: $0.47(0.18,1.21)$} & \\
\hline & & $\mathrm{N}=52 \mathrm{BRCA} 2$ cases & & & \\
\hline \multirow[t]{2}{*}{$\begin{array}{l}\text { Wu et al. } 2009 \\
\text { (USA) [37] }\end{array}$} & \multirow[t]{2}{*}{ Case-control } & $\begin{array}{l}\text { Invasive and borderline } \\
\text { ovarian cancer }\end{array}$ & $\begin{array}{l}\text { Race/ethnicity, age, education, family history } \\
\text { of ovarian cancer, menopausal status, use of } \\
\text { oral contraceptives, parity }\end{array}$ & \multirow[t]{2}{*}{$0.66(0.47,0.93)$} & \\
\hline & & $\mathrm{N}=609$ cases & & & \\
\hline \multirow{2}{*}{$\begin{array}{l}\text { Dorjgochoo T. } \\
\text { et al. } 2009 \text { (China) } \\
\text { [14] }\end{array}$} & \multirow[t]{2}{*}{ Prospective cohort } & Ovarian cancer & \multirow{2}{*}{$\begin{array}{l}\text { Age, education, age at menarche, parity, } \\
\text { breastfeeding, BMI, physical activity, smoking, } \\
\text { menopausal status, family history of cancer, } \\
\text { other contraceptive methods. }\end{array}$} & \multirow[t]{2}{*}{$1.17(0.62,2.26)$} & Cohort N=66,661 \\
\hline & & $\mathrm{N}=94$ cases & & & 76.1\% participation rate \\
\hline \multirow{4}{*}{$\begin{array}{l}\text { Nagle et al. } 2008 \\
\text { (Australia) [15] }\end{array}$} & \multirow[t]{4}{*}{ Case-control } & Invasive epithelial & \multirow{4}{*}{$\begin{array}{l}\text { Age, education, parity, and hormone } \\
\text { contraceptive use }\end{array}$} & Endometrioid: $0.4(0.3,0.7)$ & \multirow[t]{4}{*}{$47 \%$ participation rate in controls } \\
\hline & & $\begin{array}{l}\text { endometriold and clear cell } \\
\text { ovarian cancer }\end{array}$ & & Clear cell: $0.7(0.4,1.2)$ & \\
\hline & & $\mathrm{N}=142$ endometrioid cases & & & \\
\hline & & $\mathrm{N}=90$ clear cell cases & & & \\
\hline \multirow[t]{2}{*}{$\begin{array}{l}\text { Jordan et al. } 2008 \\
\text { (Australia) [10] }\end{array}$} & \multirow[t]{2}{*}{ Case-control } & $\begin{array}{l}\text { Invasive epithelial serous } \\
\text { ovarian cancer }\end{array}$ & \multirow[t]{2}{*}{$\begin{array}{l}\text { Parity, hormonal contraceptive use, history of } \\
\text { breast or ovarian cancer, age, education }\end{array}$} & \multirow[t]{2}{*}{$\begin{array}{l}\text { Serous (invasive): } 0.87 \text { (0.69- } \\
\text { 1.09) }\end{array}$} & \\
\hline & & $\mathrm{N}=627$ cases & & & \\
\hline \multirow{3}{*}{$\begin{array}{l}\text { Jordan et al. } 2007 \\
\text { (Australia) [16] }\end{array}$} & \multirow[t]{3}{*}{ Case-control } & \multirow{3}{*}{$\begin{array}{l}\text { Epithelial benign serous } \\
\text { tumors }(\mathrm{N}=230) \text { and benign } \\
\text { mucinous tumors }(\mathrm{N}=133)\end{array}$} & \multirow{3}{*}{$\begin{array}{l}\text { Age, state of residence, education, parity, } \\
\text { hormonal contraceptive use, hysterectomy, } \\
\text { smoking status }\end{array}$} & Combined: 1.04 (0.76-1.44) & \multirow{3}{*}{$\begin{array}{l}65 \% \text { participation rate in cases, } \\
47 \% \text { in controls. }\end{array}$} \\
\hline & & & & Mucinous: 1.00 (0.61-1.64) & \\
\hline & & & & Serous: 1.08 (0.75-1.57) & \\
\hline
\end{tabular}

2009 (Europe and

Canada) [13]

\begin{abstract}
Wu et al. 2009
\end{abstract}
Dorjgochoo T

et al. 2009 (China)

Nagle et al. 2008 (Australia) [15]

Jordan et al. 2008

Jordan et al. 2007

(Australia) [16]
Case-control North

Carolina Ovarian Cancer

$\mathrm{N}=201$ BRCA 1 cases

Invasive and borderline

cancer

cance

nvasive epithelial

ovarian cancer

$\mathrm{N}=142$ endometrioid cases

$=90$ clear cell cases

vasive epithelial serous

Epithelial benign serous

tumors $(\mathrm{N}=230)$ and benig

hormonal contraceptive use, hysterectom

moking status

Mucinous: 1.00 (0.61-1.64) 
Table 1 Epidemiologic Studies of the Association Between Tubal Ligation and Risk of Ovarian Cancer (Continued)

\begin{tabular}{|c|c|c|c|c|c|}
\hline \multirow[t]{2}{*}{$\begin{array}{l}\text { Tworoger et al. } \\
2007 \text { (USA) [17] }\end{array}$} & \multirow[t]{2}{*}{ Prospective cohort } & $\begin{array}{l}\text { Incident invasive epithelial } \\
\text { ovarian cancer }\end{array}$ & \multirow{2}{*}{$\begin{array}{l}\text { Age, BMI, parity, smoking history, age at } \\
\text { menarche, age at menopause, duration of } \\
\text { postmenopausal hormone use, duration of } \\
\text { oral contraceptive use }\end{array}$} & \multirow[t]{2}{*}{$0.66(0.50,0.87)$} & \multirow[t]{2}{*}{ Update of Hankinson et al. 1993} \\
\hline & & $\mathrm{N}=612$ cases & & & \\
\hline \multirow{5}{*}{$\begin{array}{l}\text { McLaughlin JR } \\
\text { et al. } 2007 \\
\text { (International) [18] }\end{array}$} & \multirow[t]{5}{*}{ Case-control } & $\begin{array}{l}\text { Invasive ovarian cancer (only } \\
\text { BRCA } 1 / 2 \text { carriers) }\end{array}$ & \multirow{5}{*}{$\begin{array}{l}\text { Age, mutation type, country of residence, } \\
\text { parity, breastfeeding, oral contraceptive use, } \\
\text { ethnicity. }\end{array}$} & $\begin{array}{l}\text { BRCA } 1+2 \text { carriers: } 0.78(0.60, \\
1.00)\end{array}$ & \multirow{5}{*}{$\begin{array}{l}\text { Includes prevalent and incident } \\
\text { cases. Results similar when } \\
\text { restricted to women interviewed } \\
\text { within } 3 \text { years of diagnosis. }\end{array}$} \\
\hline & & & & BRCA1: $0.80(0.59,1.08)$ & \\
\hline & & $\mathrm{N}=799$ cases & & \multirow[t]{3}{*}{ BRCA2: $0.63(0.34,1.15)$} & \\
\hline & & BRCA1 N=670 BRCA2 N=128 & & & \\
\hline & & $\mathrm{BRCA} 1 / 2 \mathrm{~N}=1$ & & & \\
\hline \multirow{2}{*}{$\begin{array}{l}\text { Modugno et al. } \\
2004 \text { (USA) [9] }\end{array}$} & \multirow[t]{2}{*}{ Pooled case-control } & Epithelial ovarian cancer & \multirow{2}{*}{$\begin{array}{l}\text { Study site, age, family history, duration of oral } \\
\text { contraceptive use, parity }\end{array}$} & \multirow[t]{2}{*}{$0.63(0.54,0.73)$} & \multirow[t]{2}{*}{ Pooled analysis from four studies. } \\
\hline & & $\mathrm{N}=2098$ cases & & & \\
\hline \multirow{4}{*}{$\begin{array}{l}\text { Kjaer et al. 2004 } \\
\text { (Denmark) [19] }\end{array}$} & \multirow{4}{*}{$\begin{array}{l}\text { Population-based follow- } \\
\text { up study }\end{array}$} & Invasive ovarian cancer and & \multirow[t]{4}{*}{ Age and calendar year } & Invasive: $0.82(0.6,1.0)$ & \multirow{4}{*}{$\begin{array}{l}\text { Observed number of cancer } \\
\text { cases in cohort of women who } \\
\text { underwent tubal ligation was } \\
\text { compared to the expected } \\
\text { number of cases based on the } \\
\text { age and calendar year specific } \\
\text { rates from the Danish Cancer } \\
\text { Registry. }\end{array}$} \\
\hline & & borderline ovarian tumor & & \multirow[t]{3}{*}{ Borderline: $0.82(0.5,1.3)$} & \\
\hline & & $\mathrm{N}=75$ invasive cases & & & \\
\hline & & $\mathrm{N}=21$ borderline cases & & & \\
\hline \multirow[t]{3}{*}{$\begin{array}{l}\text { McGuire et al. } \\
2004 \text { (USA) [20] }\end{array}$} & \multirow[t]{3}{*}{ Case-control } & $\begin{array}{l}\text { Invasive epithelial ovarian } \\
\text { cancer }\end{array}$ & \multirow[t]{3}{*}{ Age, parity, duration of OC use, race/ethnicity } & $\begin{array}{l}\text { BRCA } 1 \text { carriers: } 0.68(0.25 \\
1.90)\end{array}$ & \\
\hline & & $\mathrm{N}=36 \mathrm{BRCA} 1$ cases & & Noncarriers: $0.65(0.45,0.95)$ & \\
\hline & & $\mathrm{N}=381$ noncarrier cases & & & \\
\hline \multirow{2}{*}{$\begin{array}{l}\text { Pike et al. } 2004 \\
\text { (Los Angeles, USA) } \\
\text { [21] }\end{array}$} & \multirow[t]{2}{*}{ Case-control } & Invasive ovarian cancer & \multirow{2}{*}{$\begin{array}{l}\text { Age, ethnicity, SES, education, family history of } \\
\text { ovarian cancer, use of talc, BMl, parity, age at } \\
\text { last birth, number of incomplete pregnancies, } \\
\text { OC use, menopausal status, age at } \\
\text { menopause, hormone replacement therapy }\end{array}$} & \multirow[t]{2}{*}{$0.82(0.53-1.26)$} & \\
\hline & & $\mathrm{N}=477$ cases & & & \\
\hline \multirow[t]{2}{*}{$\begin{array}{l}\text { Rutter et al. } 2003 \\
\text { (Israel) [23] }\end{array}$} & \multirow[t]{2}{*}{ Case-control } & $\begin{array}{l}\text { Invasive epithelial ovarian } \\
\text { cancer or primary peritoneal } \\
\text { cancer }\end{array}$ & \multirow[t]{2}{*}{$\begin{array}{l}\text { Age, ethnicity, parity, years of oral } \\
\text { contraceptive use }\end{array}$} & \multirow[t]{2}{*}{$0.70(0.42,1.18)$} & \multirow[t]{2}{*}{$\begin{array}{l}\text { Participation rate was } 79 \% \text { for } \\
\text { case patients and } 66 \% \text { for } \\
\text { controls. }\end{array}$} \\
\hline & & $\mathrm{N}=1124$ cases & & & \\
\hline \multirow{3}{*}{$\begin{array}{l}\text { Wittenberg et al. } \\
1999 \text { (USA) [24] }\end{array}$} & \multirow[t]{3}{*}{ Case-control } & Mucinous and non-mucinous & Age at diagnosis, parity, duration of $\mathrm{OC}$ use & Mucinous: $0.4(0.1,1.9)$ & $64 \%$ participation rate in cases, \\
\hline & & epithellal ovarian cancer & & Non-mucinous: $0.6(0.3,1.1)$ & $\begin{array}{l}\text { borderline and invasive. } \\
\text { borls both }\end{array}$ \\
\hline & & $\mathrm{N}=279$ non-mucinous cases & & & \\
\hline $\begin{array}{l}\text { Kreiger et al, } 1997 \\
\text { (Canada) [25] }\end{array}$ & Historical cohort study & $\begin{array}{l}\text { Invasive and borderline } \\
\text { ovarian cancer }\end{array}$ & Age, calendar year, length of follow-up & $0.57 p<0.001$ & $\begin{array}{l}\text { Calculated observed over } \\
\text { expected events. }\end{array}$ \\
\hline & & $\begin{array}{l}\mathrm{N}=108 \text { observed cases in } \\
\text { tubal ligation subcohort }\end{array}$ & & & $\begin{array}{l}\text { Sensitivity analysis excluding } \\
\text { borderline malignancies similar. }\end{array}$ \\
\hline
\end{tabular}

cases in cohort of women who

underwent tubal ligation was

number of cases based on the endar year specific es from the Danish Cancer

$\mathrm{N}=36$ BRCA1 cases

noncarrier cases

vasive ovarian cance A arian cancer, use of talc, BMl, parity, age at ast birth, number of incomplete pregnancies,

Participation rate was 79\% for case patients and $66 \%$ for 
Table 1 Epidemiologic Studies of the Association Between Tubal Ligation and Risk of Ovarian Cancer (Continued)

\begin{tabular}{|c|c|c|c|c|c|}
\hline \multirow{2}{*}{$\begin{array}{l}\text { Green, Purdie, } \\
\text { et al. } 1997 \\
\text { (Australia) [26] }\end{array}$} & \multirow[t]{2}{*}{ Case-control } & $\begin{array}{l}\text { Incident, primary epithelial } \\
\text { ovarian cancer }\end{array}$ & \multirow[t]{2}{*}{$\begin{array}{l}\text { Age, education, BMl, parity, OC duration, } \\
\text { smoking, family history of ovarian cancer }\end{array}$} & \multirow[t]{2}{*}{$0.61(0.46,0.85)$} & \multirow[t]{2}{*}{$\begin{array}{l}90 \% \text { participation rate in cases, } \\
73 \% \text { in controls. }\end{array}$} \\
\hline & & $\mathrm{N}=824$ cases & & & \\
\hline $\begin{array}{l}\text { Cornelison et al } \\
1997 \text { (USA) [27] }\end{array}$ & Case-control & Ovarian cancer $\mathrm{N}=300$ cases & $\begin{array}{l}\text { Age, SES, marital status, parity, age at first } \\
\text { pregnancy, age at menarche, age at } \\
\text { menopause, irregular menses, breast-feeding } \\
\text { duration, BMI, OC use }\end{array}$ & $0.52(0.31,0.85)$ & $\begin{array}{l}\text { Patient controls with no } \\
\text { malignancy or ovarian disease. }\end{array}$ \\
\hline \multirow{2}{*}{$\begin{array}{l}\text { Miracle-McMahill, } \\
\text { et al. } 1997 \text { (USA) } \\
\text { [28] }\end{array}$} & \multirow[t]{2}{*}{ Prospective Cohort Study } & Ovarian cancer mortality & \multirow{2}{*}{$\begin{array}{l}\text { Age, race, BMI, education, family history of } \\
\text { ovarian cancer, family history of breast ca, } \\
\text { parity, marital status, age at menarche, OC } \\
\text { use, ERT, age at menopause, miscarriages } \\
\text { smoking status }\end{array}$} & \multirow[t]{2}{*}{$0.68(0.45,1.03)$} & \\
\hline & & $\mathrm{N}=799$ ovarian cancer deaths & & & \\
\hline \multirow{2}{*}{$\begin{array}{l}\text { Rosenblatt, et al. } \\
1996 \\
\text { (International) [29] }\end{array}$} & \multirow[t]{2}{*}{ Case-control } & $\begin{array}{l}\text { Borderline or malignant } \\
\text { epithelial ovarian cancer }\end{array}$ & \multirow[t]{2}{*}{ Age, hospital, year of interview, parity OC use } & \multirow[t]{2}{*}{$0.71(0.47,1.08)$} & \multirow{2}{*}{$\begin{array}{l}\text { No differences observed for } \\
\text { borderline and malignant } \\
\text { tumors. }\end{array}$} \\
\hline & & $\mathrm{N}=385$ cases & & & \\
\hline \multirow{3}{*}{$\begin{array}{l}\text { Risch et al. } 1996 \\
\text { (Canada) [22] }\end{array}$} & \multirow[t]{3}{*}{ Case-control } & Epithelial ovarian cancer & \multirow{3}{*}{$\begin{array}{l}\text { Age, parity, years of OC use, average lactation/ } \\
\text { pregnancy, total years of ERT, hysterectomy, } \\
\text { family history of breast cancer }\end{array}$} & \multirow[t]{3}{*}{$0.67(0.47-0.94)$} & \multirow{3}{*}{$\begin{array}{l}\text { Invasive and borderline tumors } \\
\text { included. }\end{array}$} \\
\hline & & $\mathrm{N}=450$ cases Borderline & & & \\
\hline & & $\mathrm{N}=83$ Invasive $\mathrm{N}=376$ & & & \\
\hline \multirow{2}{*}{$\begin{array}{l}\text { Nandakumar et al. } \\
1995 \text { (India) [30] }\end{array}$} & \multirow[t]{2}{*}{ Case-control } & Ovarian cancer & \multirow[t]{2}{*}{ Age, residential area, parity, age at first birth } & \multirow[t]{2}{*}{$0.25(0.08,0.78)$} & \multirow{2}{*}{$\begin{array}{l}\text { Restricted to ever-married } \\
\text { women. Hospital-based controls. }\end{array}$} \\
\hline & & $\mathrm{N}=97$ cases & & & \\
\hline \multirow{3}{*}{$\begin{array}{l}\text { Whittemore et al } \\
1992 \text { (USA) [31] }\end{array}$} & \multirow[t]{3}{*}{ Pooled case-control } & Invasive epithelial ovarian & \multirow[t]{3}{*}{ Age, study, parity, OC use } & Hospital-based studies: & \multirow{3}{*}{$\begin{array}{l}\text { Restricted to white women. } 6 \\
\text { hospital based studies and } 6 \\
\text { population-based studies. }\end{array}$} \\
\hline & & & & $\begin{array}{l}0.59(0.38,0.93) \text { Population- } \\
\text { based studies: } 0.87(0.62,1.20)\end{array}$ & \\
\hline & & $\mathrm{N}=2197$ cases & & & \\
\hline \multirow{2}{*}{$\begin{array}{l}\text { Booth et al } 1989 \\
\text { (England) [32] }\end{array}$} & \multirow[t]{2}{*}{ Case-control } & Epithelial ovarian cancer & \multirow{2}{*}{$\begin{array}{l}\text { Age, social class, gravidity, unprotected } \\
\text { intercourse }\end{array}$} & \multirow[t]{2}{*}{$0.2(0.1,0.6)$} & \multirow{2}{*}{$\begin{array}{l}\text { Cases were less than } 65 \text { years old } \\
\text { and interviewed within } 2 \text { years of } \\
\text { diagnosis. Age-matched hospital- } \\
\text { based controls. }\end{array}$} \\
\hline & & $\mathrm{N}=235$ cases & & & \\
\hline \multirow[t]{2}{*}{$\begin{array}{l}\text { Shu et al } 1989 \\
\text { (China) [33] }\end{array}$} & \multirow[t]{2}{*}{ Case-control } & $\begin{array}{l}\text { Invasive epithelial ovarian } \\
\text { cancer }\end{array}$ & $\begin{array}{l}\text { Age, education, parity, age at menarche, } \\
\text { ovarian cyst }\end{array}$ & $0.8(0.4,1.6)$ & $\begin{array}{l}89 \% \text { participation rate in cases, } \\
100 \% \text { in controls. All }<70 \text { years }\end{array}$ \\
\hline & & $\mathrm{N}=172$ cases & & & of age. \\
\hline Koch et al 1988 & Case-control & Epithelial ovarian cancer & None & $0.8(0.5,1.3)$ & $47 \%$ participation rate in controls. \\
\hline & & $\mathrm{N}=200$ cases & & & control for age in analyses. \\
\hline $\begin{array}{l}\text { Mori et al } 1988 \\
\text { (Japan) [36] }\end{array}$ & Case-control & $\begin{array}{l}\text { Primary epithelial ovarian } \\
\text { cancer }\end{array}$ & $\begin{array}{l}\text { Age, parity, marital status, number of induced } \\
\text { abortions }\end{array}$ & $0.5(0.25,1.00)$ & $\begin{array}{l}\text { Controls were hospital in-patients } \\
\text { with gynecological complaints }\end{array}$ \\
\hline & & $\mathrm{N}=110$ cases & & & $\begin{array}{l}\text { Other than ovarian cancer and } \\
\text { OB/GYN outpatients without a } \\
\text { malignant ovarian disorder. } \\
100 \% \text { participation rate in cases } \\
\text { and controls. }\end{array}$ \\
\hline
\end{tabular}

\section{$\mathrm{N}=385$ cases}

$\mathrm{N}=450$ cases Borderline

of OC use, average lactation/ pregnancy, total years of ERT, hysterectomy,

\section{Ovarian cancer}

\section{Whittemore et a}

Booth et al 1989

Shu et al 1989

Koch et al 1988

Mori et al 1988 
Table 1 Epidemiologic Studies of the Association Between Tubal Ligation and Risk of Ovarian Cancer (Continued)

\begin{tabular}{llll}
\hline Koch et al. 1984 & Retrospective cohort & Ovarian cancer N=4 cases & Age, nulliparity
\end{tabular}

(Canada) [35]

Population who underwent tuba

ere mental patients.

$34 \%$ were lost to follow-up.

Many underwent the procedure

at young ages (i.e. 10-19)

Expected rates calculated from a

previous retrospective study.

Abbreviations: OR, odds ratio; RR, relative risk; SIR, standardized incidence ratio; OC, oral contraceptive; BMI, body mass index; SES, socio-economic status; ERT, estrogen replacement therapy. 


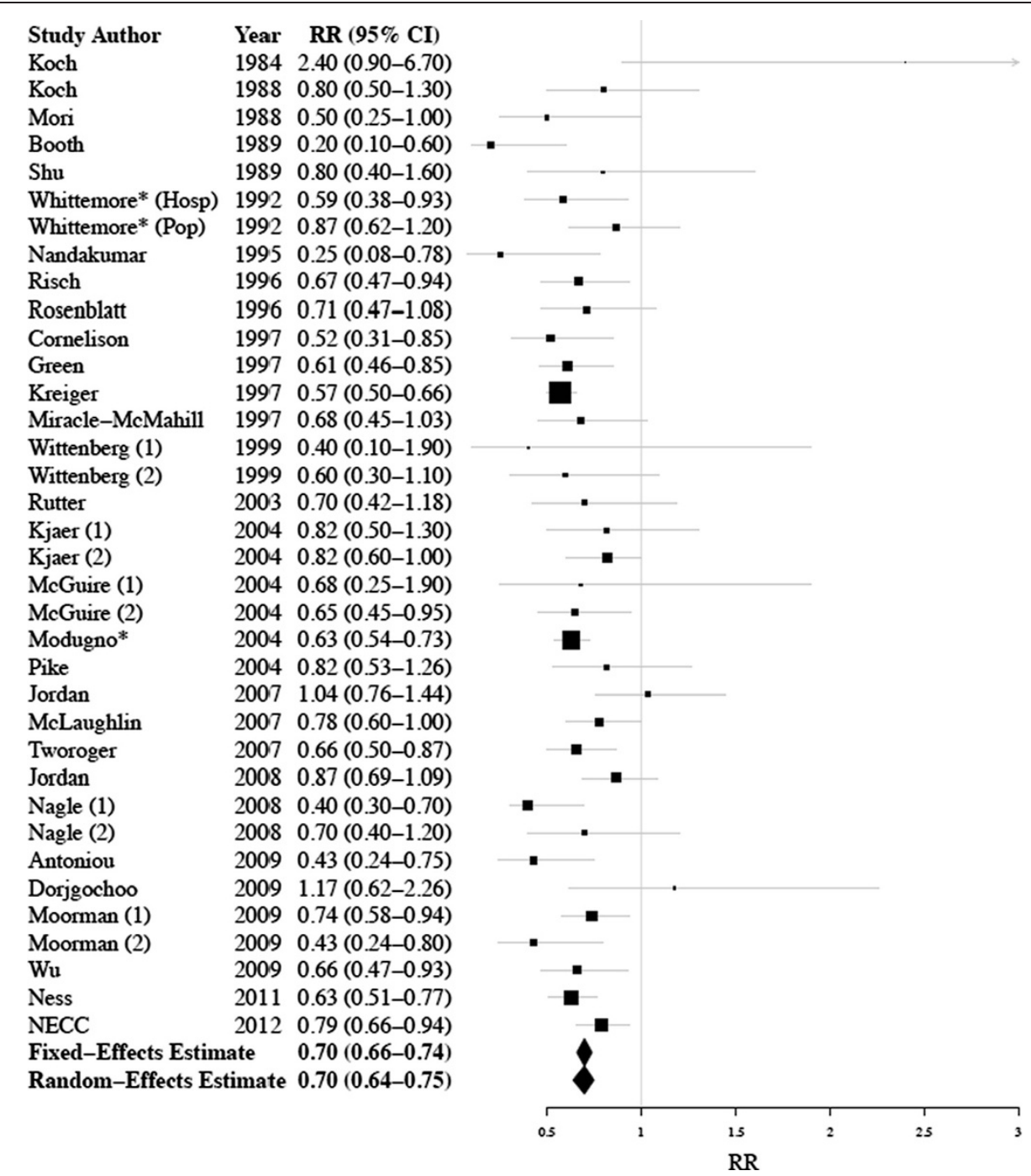

Figure $\mathbf{2}$ Forest plot for $\mathbf{3 0}$ studies of the association between tubal ligation and ovarian cancer risk. Forest plot summarizing individual effect estimates from 30 studies [9-37] contributing to summary effect estimates describing the association between tubal ligation and ovarian cancer risk. Black boxes mark the effect estimate for individual studies and the size of the black boxes represent the weight of individual studies in the summary estimate; horizontal gray lines demonstrate the width of the $95 \% \mathrm{Cls}$ associated with each individual study; the black diamonds represent summary effect estimates; stars indicate pooled studies.

participants (i.e., USA or non-USA) $(\mathrm{P}>0.05)$ (Table 2). Interestingly, the relative risk among BRCA carriers $(\mathrm{RR}=0.64,95 \% \mathrm{CI}: 0.43-0.96)$ was similar to the relative risk among population-based studies $(\mathrm{RR}=0.70,95 \% \mathrm{CI}$ : 0.64-0.76) (Table 2). Overall, we found that if any single study was removed from the meta-analysis, the effect estimate did not change substantially (data not shown). In addition, we found no evidence of publication bias using either the Begg $(P=0.12)$ or the Egger $(P=0.22)$ method for assessing bias.

Eight of the studies examined years since tubal ligation. In a meta-regression of six of these studies, we did not observe a difference in the relative risk of ovarian cancer between women who had a tubal ligation less than 10 years ago (summary $\mathrm{RR}=0.69,95 \% \mathrm{CI}: 0.59$, $0.79)$ and those women who had a tubal ligation 10 or more years ago (summary $\mathrm{RR}=0.68,95 \% \mathrm{CI}$ : $0.54,0.87$ ) $(P$-heterogeneity $=0.78)($ Table 2$)$. Of the other studies, a prospective cohort study of ovarian cancer mortality reported tubal ligation to be associated with a reduced risk for women who had the procedure within 20 years, with a smaller non-significant reduced risk for those who had the procedure 20 or more years ago.[19] However, a prospective cohort study based in China observed a non-significant increase in risk that was similar for both women who had a tubal ligation less than 33 years ago and women who had a tubal ligation 33 or more years ago [14]. 
Table 2 Summary relative risks for tubal ligation and ovarian cancer by selected characteristics

\begin{tabular}{|c|c|c|}
\hline & $\begin{array}{c}\text { Number of } \\
\text { contributing studies }\end{array}$ & $\begin{array}{c}\text { Random-effects } \\
\text { RR }(95 \% \mathrm{Cl}) \\
\end{array}$ \\
\hline Study design & 30 studies & \\
\hline Cohort study & & $0.67(0.50,0.90)$ \\
\hline Case-control study & & $0.70(0.63,0.75)$ \\
\hline Other study design & & $0.95(0.63,1.43)$ \\
\hline BRCA status & 30 studies & \\
\hline BRCA positive & & $0.64(0.43,0.96)$ \\
\hline General population & & $0.70(0.64,0.76)$ \\
\hline Geographic location & 30 studies & \\
\hline US & & $0.68(0.63,0.73)$ \\
\hline Non-US & & $0.71(0.61,0.82)$ \\
\hline Histologic subtype & 11 studies & \\
\hline Serous & & $0.75(0.65,0.88)$ \\
\hline Endometrioid & & $0.45(0.33,0.61)$ \\
\hline Mucinous & & $0.88(0.70,1.09)$ \\
\hline Clear cell & & $0.72(0.55,0.94)$ \\
\hline Other & & $0.80(0.63,1.01)$ \\
\hline Age at tubal ligation & 7 studies & \\
\hline$<35$ years of age & & $0.69(0.59,0.81)$ \\
\hline $35+$ years of age & & $0.79(0.68,0.92)$ \\
\hline Years since tubal ligation & 6 studies & \\
\hline$<10$ years & & $0.69(0.59,0.79)$ \\
\hline $10+$ years & & $0.68(0.54,0.87)$ \\
\hline
\end{tabular}

Nine studies examined age at tubal ligation on ovarian cancer risk. In a meta-regression of seven of these studies, the relative risk for ovarian cancer was non-significantly lower among women who had a tubal ligation when they were younger than 35 (summary $\mathrm{RR}=0.69,95 \% \mathrm{CI}$ : 0.59 , 0.81 ) compared to at 35 years of age or older (summary $\mathrm{RR}=0.79,95 \% \mathrm{CI}: 0.68,0.92)$, although the difference was not statistically significant $(P$-for-heterogeneity $=0.22)$ (Table 2). In addition, the Shanghai Women's Health Study noted a non-significant increase in ovarian cancer risk only among women who were less than 30 when they underwent the procedure and no association among those aged 30 or more at time of surgery [14]. In a historical cohort study, tubal ligation was associated with a reduced risk of ovarian cancer among women aged 25-44 at time of the procedure $(R R=0.54, p<0.001)$, but not among women aged $45-64$ at the time of their tubal ligation $(\mathrm{RR}=1.18, \mathrm{p}=0.68)[25]$.

Eleven studies reported effect estimates by at least one histologic subtype. In a meta-analysis regression we observed that the association was stronger for endometrioid tumors compared to serous tumors $(P<0.01)$. The summary RR for serous tumors was 0.75 (95\%CI:
$0.65,0.88$ ) compared to 0.45 (95\%CI: $0.33,0.61$ ) for endometrioid tumors. The summary RRs for mucinous (summary RR $=0.88,95 \% \mathrm{CI}: 0.70,1.09$ ), clear cell (summary $\mathrm{RR}=0.72,95 \% \mathrm{CI}: 0.55,0.94)$, and other tumor types (summary RR $=0.80,95 \% \mathrm{CI}$ : $0.63,1.01$ ) did not significantly differ from serous tumors $(\mathrm{p}>0.05)$.

\section{Hysterectomy}

The study-specific RRs for ovarian cancer associated with hysterectomy (with or without unilateral oophorectomy) ranged from 0.06 to 1.91 (Table 3 ). The summary RR was 0.74 (95\%CI: 0.65, 0.84), demonstrating a statistically significant inverse association between hysterectomy and ovarian cancer (Figure 3). When we restricted to nine studies that reported effect estimates for invasive ovarian cancer, the association was similar (summary $\mathrm{RR}=0.81$; 95\%CI: $0.68,0.97)$. We also calculated summary estimates for simple hysterectomy and hysterectomy with unilateral oophorectomy (Table 4). We observed that the reduced risk of ovarian cancer associated with hysterectomy with unilateral oophorectomy $(\mathrm{RR}=0.60,95 \% \mathrm{CI}: 0.47-0.78)$ was similar to the reduced risk associated with simple hysterectomy $(R R=0.62,95 \%$ CI: 0.49-0.79). We examined the contribution of other study characteristics to the heterogeneity between studies, since the p-heterogeneity $<0.01$. We did not observe evidence for statistically significant heterogeneity by study type (i.e., case-control, cohort, other) or geographic location (i.e., USA vs non-USA) $(P>0.05)$ (Table 4). Overall, if any single study was removed from the meta-analysis, the effect estimate did not change substantially (data not shown). We did note evidence of publication bias using the Egger $(P=0.01)$ method for assessing bias, but not for the Begg method $(\mathrm{P}=0.11)$.

Eight studies examined years since hysterectomy and ovarian cancer risk. In a meta-regression of seven of these studies, the RR of ovarian cancer between women who had the procedure 10 or more years ago was slightly lower compared to women who had a hysterectomy less than 10 years ago (summary $R R=0.69,95 \%$ CI: $0.60,0.79$ and summary $\mathrm{RR}=0.77,95 \% \mathrm{CI}: 0.66$, 0.89 respectively) (P-heterogeneity $=0.33)$. In addition, a hospital-based case-control study reported an inverse association among women who underwent the procedure more than five years ago $(\mathrm{RR}=0.37,95 \% \mathrm{CI}$ : $0.11-1.24)$, but no association among those who had a hysterectomy within five years $(\mathrm{RR}=1.04,95 \% \mathrm{CI}$ : 0.37-2.90) [29]. Five studies examined age at hysterectomy on ovarian cancer risk, three dichotomized at age 40 and two at age 45 . In a meta-regression, hysterectomy was more strongly inversely associated with ovarian cancer among women who were younger than 40 or 45 at surgery compared to 40 or 45 years of age or older, however the $\mathrm{p}$ for heterogeneity was not 
Table 3 Epidemiologic Studies of the Association Between Hysterectomy and Risk of Ovarian Cancer

\begin{tabular}{|c|c|c|c|c|c|}
\hline Author (Country) & Study Design & Case definition & Covariates & OR, RR, or SIR $(95 \% \mathrm{Cl})$ & Comments \\
\hline \multirow{2}{*}{$\begin{array}{l}\text { NECC } 2012 \text { (USA) } \\
\text { [Personal } \\
\text { communication } \\
\text { with Dr. Daniel } \\
\text { Cramer] }\end{array}$} & \multirow[t]{2}{*}{ Case-control } & $\begin{array}{l}\text { Borderline and invasive ovarian } \\
\text { cancer }\end{array}$ & \multirow{2}{*}{$\begin{array}{l}\text { age, study center, BMI, study phase, } \\
\text { smoking, family history of ovarian and } \\
\text { breast cancers, talc use, OC use, parity, } \\
\text { breast feeding, age at menarche, post- } \\
\text { menopausal status, use of post-menopausal } \\
\text { hormones, tubal ligation }\end{array}$} & Hysterectomy only: 1.10 (0.83-1.46) & \multirow{4}{*}{$\begin{array}{l}\text { NECC } 2012 \text { (USA) [Personal } \\
\text { communication with Dr. Daniel } \\
\text { Cramer] }\end{array}$} \\
\hline & & $N=2076$ & & $\begin{array}{l}\text { Hysterectomy with unilateral } \\
\text { oophorectomy: } 0.68 \text { (0.46-0.99) }\end{array}$ & \\
\hline \multirow{2}{*}{$\begin{array}{l}\text { Annegers et al. } \\
1979 \text { (USA) [38] }\end{array}$} & \multirow{2}{*}{$\begin{array}{l}\text { Case-control } \\
\text { (Rochester Project) }\end{array}$} & \multirow{2}{*}{$\begin{array}{l}\text { Epithelial ovarian cancer } \mathrm{N}=116 \\
\text { cases }\end{array}$} & \multirow[t]{2}{*}{ Controls matched on age and residence } & Hysterectomy only: $0.36(0.10-0.73)$ & \\
\hline & & & & $\begin{array}{l}\text { Hysterectomy with unilateral } \\
\text { oophorectomy: } 0.06 \text { (0.004-0.98) }\end{array}$ & \\
\hline \multirow{4}{*}{$\begin{array}{l}\text { Antoniou et al. } \\
2009 \text { (Europe and } \\
\text { Canada) [13] }\end{array}$} & \multirow{4}{*}{ Retrospective Cohort } & $\begin{array}{l}\text { Ovarian cancer (only BRCA } 1 / 2 \\
\text { carriers) }\end{array}$ & \multirow[t]{4}{*}{ Age, duration of $O C$ use, parity } & \multirow{2}{*}{$\begin{array}{l}\text { Hysterectomy with or without } \\
\text { unilateral oophorectomy: BRCA 1/2: } \\
0.59(0.22,1.57)\end{array}$} & $\begin{array}{l}\text { Includes prevalent and incident } \\
\text { cases. }\end{array}$ \\
\hline & & $\mathrm{N}=201 \mathrm{BRCA} 1$ cases & & & \multirow{3}{*}{$\begin{array}{l}\text { Mean difference between age at } \\
\text { diagnosis and interview: } 6.7 \text { years }\end{array}$} \\
\hline & & \multirow[t]{2}{*}{$\mathrm{N}=52 \mathrm{BRCA} 2$ cases } & & BRCA1:0.68 $(0.22,2.12)$ & \\
\hline & & & & BRCA2: $0.35(0.08,1.58)$ & \\
\hline \multirow{2}{*}{$\begin{array}{l}\text { Beard et al. } 2000 \\
\text { (USA) [40] }\end{array}$} & \multirow{2}{*}{$\begin{array}{l}\text { Case-control } \\
\text { (Rochester Project) }\end{array}$} & Invasive epithelial ovarian cancer & \multirow[t]{2}{*}{ Controls matched on age and provider } & \multirow{2}{*}{$\begin{array}{l}\text { Hysterectomy with or without } \\
\text { unilateral oophorectomy: } 0.5 \\
(0.2-0.96)\end{array}$} & \\
\hline & & $\mathrm{N}=103$ cases & & & \\
\hline \multirow{2}{*}{$\begin{array}{l}\text { Booth et al } 1989 \\
\text { (England) [32] }\end{array}$} & \multirow[t]{2}{*}{ Case-control } & Epithelial ovarian cancer & \multirow[t]{2}{*}{ Age and social class } & Hysterectomy only: $0.2(0.1-0.4)$ & \multirow{2}{*}{$\begin{array}{l}\text { Cases less than } 65 \text { years old and } \\
\text { diagnosed within } 2 \text { years. Age- } \\
\text { matched hospital-based controls. }\end{array}$} \\
\hline & & $\mathrm{N}=235$ cases & & $\begin{array}{l}\text { Hysterectomy with unilateral } \\
\text { oophorectomy: } 0.4(0.1-1.1)\end{array}$ & \\
\hline \multirow{2}{*}{$\begin{array}{l}\text { Braem et al. } 2010 \\
\text { (Netherlands) [41] }\end{array}$} & \multirow{2}{*}{$\begin{array}{l}\text { Case-cohort study } \\
\text { (Netherlands Cohort } \\
\text { Study) }\end{array}$} & Invasive epithelial ovarian cancer & \multirow[t]{2}{*}{ Age, OC use, parity } & \multirow{2}{*}{$\begin{array}{l}\text { Hysterectomy with or without } \\
\text { unilateral oophorectomy: } 0.50 \\
(0.34-0.72)\end{array}$} & \multirow{4}{*}{$\begin{array}{l}\text { All women presumed to be } \\
\text { postmenopausal }\end{array}$} \\
\hline & & $\mathrm{N}=375$ & & & \\
\hline \multirow[t]{2}{*}{$\begin{array}{l}\text { Chiaffarino et al. } \\
2005 \text { (Italy) [42] }\end{array}$} & \multirow[t]{2}{*}{$\begin{array}{l}\text { Multi-center case- } \\
\text { control study }\end{array}$} & $\begin{array}{l}\text { Incident invasive epithelial } \\
\text { ovarian cancer }\end{array}$ & \multirow[t]{2}{*}{$\begin{array}{l}\text { Age, center, education, parity, OC use, family } \\
\text { history of ovarian and breast cancer }\end{array}$} & \multirow{2}{*}{$\begin{array}{l}\text { Hysterectomy only: } 0.6(0.4-0.9) \\
\text { Hysterectomy and unilateral } \\
\text { oophorectomy: } 0.6(0.3-1.1)\end{array}$} & \\
\hline & & $\mathrm{N}=1031$ cases & & & \\
\hline \multirow{2}{*}{$\begin{array}{l}\text { Green, Purdie, } \\
\text { et al. 1997 } \\
\text { (Australia) [26] }\end{array}$} & \multirow[t]{2}{*}{ Case-control } & $\begin{array}{l}\text { Incident, primary epithelial } \\
\text { ovarian cancer }\end{array}$ & \multirow[t]{2}{*}{$\begin{array}{l}\text { Age, education, BMI, parity, OC duration, } \\
\text { smoking, family history of ovarian cancer }\end{array}$} & $\begin{array}{l}\text { Hysterectomy with or without } \\
\text { unilateral oophorectomy: } 0.64\end{array}$ & $\begin{array}{l}90 \% \text { participation rate in cases, } 73 \% \\
\text { in controls. }\end{array}$ \\
\hline & & $\mathrm{N}=824$ cases & & & \\
\hline $\begin{array}{l}\text { Hankinson et al. } \\
1993 \text { (USA) [43] }\end{array}$ & Cohort study (NHS) & $\begin{array}{l}\text { Borderline and malignant } \\
\text { epithelial ovarian cancer }\end{array}$ & $\begin{array}{l}\text { Age, parity, duration of OC use, age at } \\
\text { menarche, tubal ligation, smoking status, }\end{array}$ & Hysterectomy only: 0.67 (0.45-1.00) & $90 \%$ follow-up rate \\
\hline & & $\mathrm{N}=260$ cases & & & \\
\hline $\begin{array}{l}\text { Jordan et al. } 2008 \\
\text { (Australia) [10] }\end{array}$ & Case-control & $\begin{array}{l}\text { Invasive epithelial serous ovarian } \\
\text { cancer }\end{array}$ & $\begin{array}{l}\text { Parity, hormonal contraceptive use, history } \\
\text { of breast or ovarian cancer, age, education }\end{array}$ & $\begin{array}{l}\text { Hysterectomy with or without } \\
\text { unilateral oophorectomy: }\end{array}$ & \\
\hline & & $\mathrm{N}=627$ cases & & Serous (invasive): $1.27(1.00,1.60)$ & \\
\hline $\begin{array}{l}\text { Jordan et al. } 2007 \\
\text { (Australia) [16] }\end{array}$ & Case-control & $\begin{array}{l}\text { Benign serous tumors ( } N=230) \\
\text { and benign mucinous tumors }\end{array}$ & $\begin{array}{l}\text { Age, state of residence, education, parity, } \\
\text { hormonal contraceptive use, smoking status }\end{array}$ & $\begin{array}{l}\text { Hysterectomy with or without } \\
\text { unilateral oophorectomy: }\end{array}$ & $\begin{array}{l}65 \% \text { participation rate in cases, } 47 \% \\
\text { in controls. }\end{array}$ \\
\hline & & & & Combined: 1.91 (1.38-2.66) & \\
\hline
\end{tabular}

Beard et al. 2000 Case-control

Booth et al 1989 Case-control

Braem et al. 2010 Case-cohort study etherlands Cohor

Chiaffarino et al. Multi-center case-

Green, Purdie, Case-control

et al. 1997

(Australia) [26]

Hankinson et al. Cohort study (NHS)

Jordan et al. 2008 Case-control

Jordan et al. 2007 Case-control (Australia) [16]
Hysterectomy with unilateral

Hysterectomy with or without

$0.59(0.22,1.57)$

rectomy with or without

unilateral oophorectomy: 0.5

Hysterectomy only: 0.2 (0.1-0.4)

Hysterectomy with or without Unilateral oophorectomy: 0.50

erectomy with or without unilateral oophorectomy: 0.64 $(0.48-0.85)$

$\mathrm{N}=260$ cases

Invasive epithelial serous ovarian Parity, hormonal contraceptive use, history $\mathrm{N}=627$ cases

a $(\mathrm{N}=133)$

Combined: $1.91(1.38-2.66)$ 

up. Non-hysterectomized women had $\mathrm{N}=91$ cases with total Epithelial ovarian cancer $\mathrm{N}=746$ White cases Invasive epithelial endometrioid 142 endometrioid cas Epithelial ovarian cancer (a) 0.51 unilateral (a) 
Table 3 Epidemiologic Studies of the Association Between Hysterectomy and Risk of Ovarian Cancer (Continued)

\begin{tabular}{|c|c|c|c|c|c|}
\hline & & & & $\begin{array}{l}\text { Hysterectomy with unilateral } \\
\text { oophorectomy: } 1.06 \text { (0.34-3.29) }\end{array}$ & \\
\hline & & & & Combined: 0.58 (0.27-1.28) & \\
\hline \multirow[t]{2}{*}{$\begin{array}{l}\text { Rutter et al. } 2003 \\
\text { (Israel) [23] }\end{array}$} & \multirow[t]{2}{*}{ Case-control } & $\begin{array}{l}\text { Epithelial ovarian cancer or } \\
\text { primary peritoneal cancer }\end{array}$ & \multirow[t]{2}{*}{$\begin{array}{l}\text { Age, ethnicity, parity, years of oral } \\
\text { contraceptive use }\end{array}$} & Hysterectomy only: 0.69 (0.50-0.95) & \multirow{2}{*}{$\begin{array}{l}\text { Participation rate was } 79 \% \text { for case } \\
\text { patients and } 66 \% \text { for controls. } \\
\text { Includes BRCA-specific analysis. }\end{array}$} \\
\hline & & $\mathrm{N}=1124$ cases & & $\begin{array}{l}\text { Hysterectomy with unilateral } \\
\text { oophorectomy: } 0.46(0.25-0.86)\end{array}$ & \\
\hline \multirow{3}{*}{$\begin{array}{l}\text { Whittemore et al } \\
1992 \text { (USA) [31] }\end{array}$} & \multirow{3}{*}{$\begin{array}{l}\text { Pooled case-control } \\
\text { (12 studies included) }\end{array}$} & Invasive epithelial ovarian cancer & \multirow[t]{3}{*}{ Age, study, parity, OC use } & Hysterectomy with or without & \multirow{3}{*}{$\begin{array}{l}\text { Restricted to white women. } 6 \\
\text { hospital based studies and } 6 \\
\text { population-based studies. All } \\
\text { hysterectomies performed at least } 2 \\
\text { years prior to reference date. }\end{array}$} \\
\hline & & \multirow[t]{2}{*}{$\mathrm{N}=2197$ cases } & & $\begin{array}{l}\text { unilateral oophorectomy: Hospital- } \\
\text { based studies: } 0.66(0.50-0.86)\end{array}$ & \\
\hline & & & & $\begin{array}{l}\text { Population-based studies: } 0.88 \\
(0.72-1.1)\end{array}$ & \\
\hline \multirow[t]{3}{*}{$\begin{array}{l}\text { Wittenberg et al. } \\
1999 \text { (USA) [24] }\end{array}$} & \multirow[t]{3}{*}{ Case-control } & $\begin{array}{l}\text { Mucinous and non-mucinous } \\
\text { epithelial ovarian cancer }\end{array}$ & \multirow[t]{3}{*}{ Age at diagnosis, parity, duration of OC use } & \multirow{2}{*}{$\begin{array}{l}\text { Hysterectomy with or without } \\
\text { unilateral oophorectomy: Mucinous: } \\
0.2(0.1,1.0)\end{array}$} & \multirow{3}{*}{$\begin{array}{l}64 \% \text { participation rate in cases, } 72 \% \\
\text { in controls. Included both } \\
\text { borderline and invasive. }\end{array}$} \\
\hline & & $\mathrm{N}=43$ mucinous cases & & & \\
\hline & & $\mathrm{N}=279$ non-mucinous cases & & Non-mucinous: $1.1(0.7,1.6)$ & \\
\hline $\begin{array}{l}\text { Wynder et al. } \\
1969 \text { (USA) [47] }\end{array}$ & $\begin{array}{l}\text { Case-control (Hospital } \\
\text { based) }\end{array}$ & $\begin{array}{l}\text { Epithelial ovarian cancer }(\mathrm{N}=150) \\
\text { plus miscellaneous ovarian } \\
\text { tumors }(\mathrm{N}=8)\end{array}$ & Age-matched controls & $\begin{array}{l}\text { Hysterectomy with or without } \\
\text { unilateral oophorectomy: } 0.7 \\
(0.04-1.0)\end{array}$ & \\
\hline
\end{tabular}

Abbreviations: OR, odds ratio; RR, relative risk; SIR, standardized incidence ratio; OC, oral contraceptive; BMI, body mass index; SES, socio-economic status. 


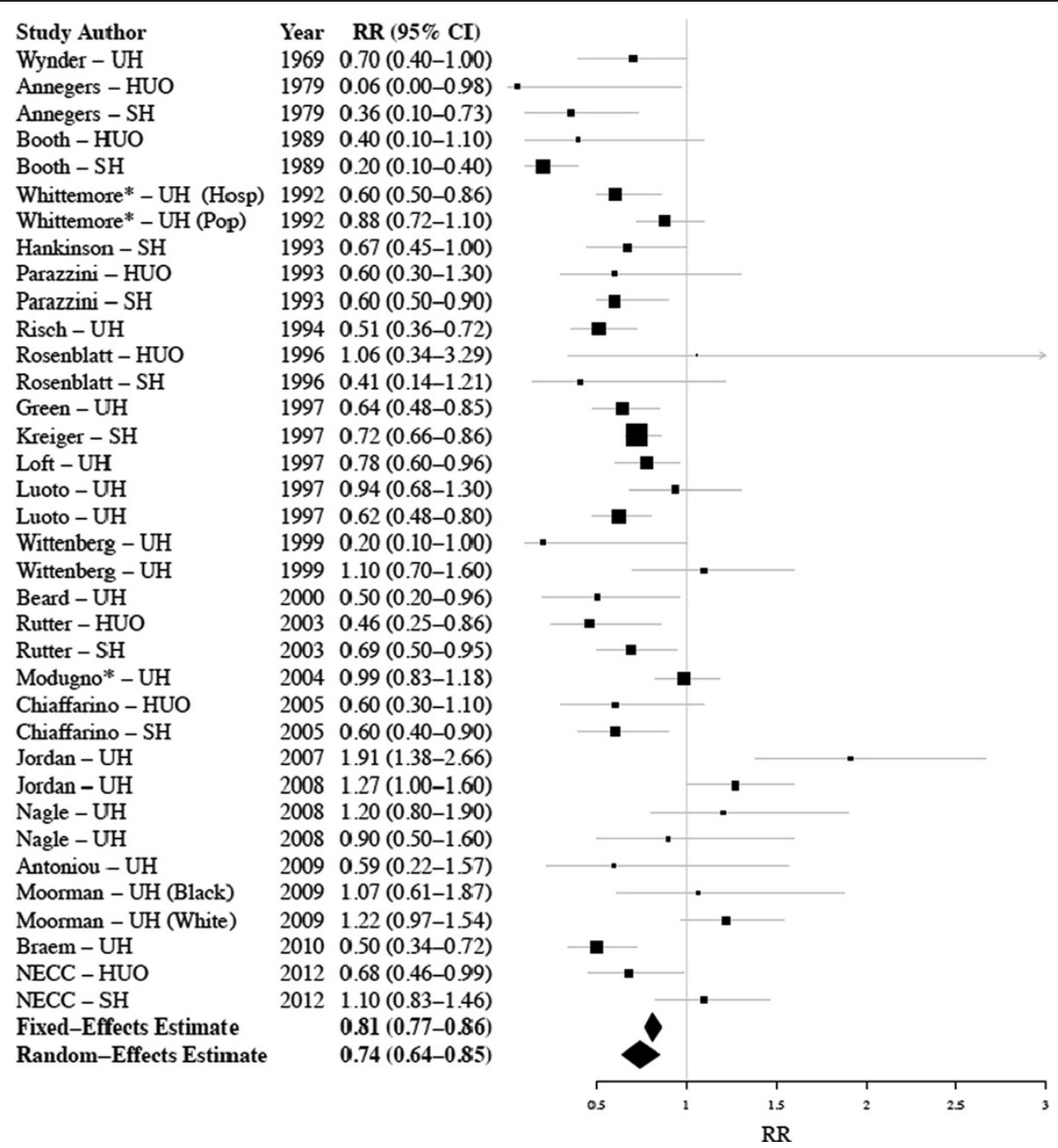

Figure 3 Forest plot for $\mathbf{2 4}$ studies of the association between hysterectomy and ovarian cancer risk. Forest plot summarizing individual effect estimates from 24 studies $[9,10,12,13,15,16,23,26,29,31,32,38-47]$ contributing to summary effect estimates describing the association between hysterectomy and ovarian cancer risk. Black boxes mark the effect estimate for individual studies and the size of the black boxes represent the weight of individual studies in the summary estimate; horizontal gray lines demonstrate the width of the $95 \%$ Cls associated with each individual study; the black diamonds represent summary effect; stars indicate pooled studies. HUO=hysterectomy with unilateral oophorectomy, SH=simple hysterectomy, UH=unknown type of hysterectomy.

statistically significant $(P$-heterogeneity $=0.29)$. The summary RR for women less than 40 or 45 years of age was $0.70(95 \% \mathrm{CI}: 0.55,0.89)$ compared to 0.83 (95\%CI: $0.72,0.96)$ for women over 40 or 45 years of age (Table 4).

\section{Discussion}

Observational epidemiologic evidence strongly suggests that there is a decreased risk of ovarian cancer among women who have had a tubal ligation or hysterectomy. We observed an approximately $26-30 \%$ reduction in ovarian cancer risk among women who had a tubal ligation or hysterectomy compared to women who never had a tubal ligation or hysterectomy, respectively. These estimates did not vary substantially by study design or population. We did not observe any significant differences in the effect estimates by years since procedure. For both hysterectomy and tubal ligation, the inverse association between these procedures and ovarian cancer risk was suggestively stronger among women who underwent the procedure at earlier ages. There was evidence that tubal ligation may be associated with a stronger reduced risk for endometrioid tumors compared to serous tumors; however this finding was based on studies with small numbers of cases of each subtype and should be interpreted cautiously.

Several mechanisms have been proposed to explain the observed inverse association between tubal ligation and hysterectomy and ovarian cancer risk. One potential explanation is a "screening effect" wherein surgeons are 
Table 4 Summary relative risks for hysterectomy and ovarian cancer by selected characteristics

\begin{tabular}{llc}
\hline & \multicolumn{1}{c}{$\begin{array}{c}\text { Number of } \\
\text { contributing studies }\end{array}$} & $\begin{array}{c}\text { Random-effects } \\
\text { RR }(95 \% \mathbf{C l})\end{array}$ \\
\hline $\begin{array}{l}\text { Study design } \\
\text { Cohort study }\end{array}$ & 24 studies & $0.73(0.63,0.85)$ \\
Case-control study & & $0.73(0.62,0.86)$ \\
Geographic location & 24 studies & \\
US & & $0.81(0.67,0.97)$ \\
Non-US & $0.70(0.59,0.84)$ \\
Type of hysterectomy & 24 studies & \\
With unilateral oophorectomy & & $0.60(0.47,0.78)$ \\
Without oophorectomy & & $0.62(0.49,0.79)$ \\
Unknown oophorectomy & & $0.83(0.71,0.98)$ \\
Age at hysterectomy & 5 studies & \\
$<40 / 45$ years of age & & $0.70(0.55,0.89)$ \\
$40 / 45+$ years of age & & $0.83(0.72,0.96)$ \\
Years since hysterectomy & 7 studies & \\
$<10$ years & & $0.69(0.60,0.79)$ \\
$10+$ years & & $0.77(0.66,0.89)$ \\
\hline
\end{tabular}

able to visualize abnormal changes in the ovaries during tubal sterilizations or hysterectomies and remove premalignant lesions. If the inverse association was solely due to screening of the ovaries, these procedures would be associated with a lower risk for only a few years after the surgery; however this was not supported in our analysis as there was a strong inverse association even more than 10 years after surgery. Another potential mechanism is that tubal ligation and hysterectomy protect the ovary from carcinogens, such as talc, or inflammatory agents such as retrograde menstruation or endometriosis ascending the genital tract. Green et al. reported that ovarian cancer risk was highest among women who used talc and did not have a tubal ligation or hysterectomy and lowest among women who had surgical sterilization, but did not use talc [26]. However, in the Nurses' Health Study (NHS), there was no variation in RR estimates of tubal ligation and ovarian cancer by talc use, and in a large case-control study, the inverse association of tubal ligation and hysterectomy was limited to non-talc users, contrary to the ascending carcinogen hypothesis $[43,50]$.

Ovarian cancer risk may be altered by decreased blood supply to the ovary after surgery resulting in a decrease in estrogen production. However, while some studies have observed decreases in hormone levels after tubal ligation or hysterectomy, [51-53] others have not $[54,55]$. This mechanism may only apply to procedures that cause substantial damage to the surrounding tissue. In the NHS, women who had undergone tubal ligation during the time period when the unipolar electrocautery method was commonly used had a reduced risk of breast cancer [56]. However, tubal ligation was not associated with breast cancer risk during other periods when methods that caused less tissue destruction were common. To our knowledge, only one study examined ovarian cancer risk by type of tubal ligation and observed a lower risk irrespective of technique [26]. However this analysis was based on only 20 cases and 58 controls and thus had limited power. Lastly, several cancers, including ovarian cancers, over-express the surface glycoprotein MUC1. It has been hypothesized that women who have undergone events that trigger an immune response to MUC1 have a decreased risk of ovarian cancer [4]. A recent study reported higher anti-MUC1 antibodies were associated with a decreased risk of ovarian cancer among women less than 64 years of age [57]. In the same study, women who had undergone a tubal ligation had higher mean levels of anti-MUC1 antibodies compared to women who had not undergone a tubal ligation; however there were no differences in antibodies levels by hysterectomy status [57]. Further research is needed to determine the associations between surgical procedures, anti-MUC1 antibodies, and subsequent ovarian cancer risk.

Our analysis has several limitations. Not all studies reported whether cases were restricted to invasive ovarian cancer., however when we restricted to studies that reported effect estimates for invasive ovarian cancer the summary RRs were very similar. Few studies reported effect estimates by surgical characteristics or histological subtype of ovarian cancer. In addition, when reported, these stratum-specific estimates were often based on small numbers of exposed cases. To pool effect estimates for analysis of age at and years since tubal ligation, we created very broad categories (e.g., age at tubal ligation $<35$ years, $\geq 35$ years; hysterectomy $<10$ years ago, $\geq 10$ years ago), which may obscure important effects. Some of the studies in the meta-analysis included both prevalent as well as incident ovarian cancer cases and the case definition for one study was ovarian cancer mortality. If tubal ligation or hysterectomy were associated with survival after ovarian cancer diagnosis then the inclusion of prevalent cases may bias the effect estimates. However, a recent systematic review did not support an association between tubal ligation or hysterectomy and survival from ovarian cancer [58].

In summary, we observed a consistent inverse association of tubal ligation and hysterectomy on ovarian cancer risk that may be causal. We did not detect differences by study design, study population, or years since the procedure, although our statistical power in these analyses was somewhat limited. While gynecologic surgery may be a potential prevention strategy for women at high risk of ovarian cancer, additional research is needed to determine 
whether the effect of tubal ligation and hysterectomy on ovarian cancer risk differs by individual and surgical characteristics as well as considering the potential negative health effects of these procedures. Additional research also is needed to further understand the mechanisms behind these reduced risks.

\section{Additional file}

Additional file 1: Table S1, Table S2, Table S3, Table S4, Table S5.

Epidemiologic Studies of the Association Between Tubal Ligation and

Risk of Ovarian Cancer by Years Since Procedure. Epidemiologic Studies of the Association Between Tubal Ligation and Risk of Ovarian Cancer by Age at Procedure. Epidemiologic Studies of the Association Between Tubal Ligation and Risk of Ovarian Cancer by Histological Subtype. Epidemiologic Studies of the Association Between Hysterectomy and Risk of Ovarian Cancer by Years Since Procedure. Epidemiologic Studies of the Association Between Hysterectomy and Risk of Ovarian Cancer by Age at Procedure [59].

\section{Competing interests}

The authors declare that they have no competing interests.

\section{Acknowledgements}

This study was supported by the National Institutes of Health grants P01 CA 87969, T32 CA 09001, P50 CA 105009, R03 CA 143918.

\section{Author details}

'Channing Laboratory, Department of Medicine, Brigham and Women's Hospital and Harvard Medical School, Boston, MA, USA. ${ }^{2}$ Department of Epidemiology, Harvard School of Public Health, Boston, MA, USA. ${ }^{3}$ Channing Laboratory, 181 Longwood Ave., 3rd Floor, Boston, MA 02115, USA.

\section{Authors' contributions}

MSR participated in the design of the study, conducted the literature search for all tubal ligation articles, extracted data, analyzed the data and authored the manuscript. MAM conducted the literature search for all hysterectomy articles and extracted data. SST participated in the design of the study, reviewed the data extracted, and helped draft the manuscript. All authors read and approved the final manuscript.

Received: 13 September 2011 Accepted: 15 May 2012 Published: 15 May 2012

\section{References}

1. Society AC: Cancer facts \& Figures 2008. 2008. In.

2. Moorman PG, Schildkraut JM, Calingaert B, Halabi S, Vine MF, Berchuck A: Ovulation and ovarian cancer: a comparison of two methods for calculating lifetime ovulatory cycles (United States). Cancer Causes Control 2002, 13(9):807-811.

3. Lukanova A, Kaaks R: Endogenous hormones and ovarian cancer: epidemiology and current hypotheses. Cancer Epidemiol Biomarkers Prev 2005, 14(1):98-107. doi:14/1/98.

4. Cramer DW, Titus-Ernstoff L, McKolanis JR, Welch WR, Vitonis AF, Berkowitz RS, Finn OJ: Conditions associated with antibodies against the tumorassociated antigen MUC1 and their relationship to risk for ovarian cancer. Cancer Epidemiol Biomarkers Prev 2005, 14(5):1125-1131. doi:14/5/ 1125 10.1158/1055-9965.EPI-05-0035.

5. DerSimonian R, Laird N: Meta-analysis in clinical trials. Control Clin Trials 1986, 7(3):177-188. doi:0197-2456(86)90046-2.

6. Begg CB, Mazumdar M, Egger M, Davey Smith G, Schneider M, Minder C, DerSimonian R, Laird N, Lau J, loannidis JP, Schmid CH: Operating characteristics of a rank correlation test for publication bias Bias in meta-analysis detected by a simple, graphical test Meta-analysis in clinical trials Summing up evidence: one answer is not always enough. Biometrics 1994, 50(4):1088-1101.

7. Egger M, Davey Smith G, Schneider M, Minder C: Bias in meta-analysis detected by a simple, graphical test. BMJ 1997, 315(7109):629-634.
8. Lau J, loannidis JP, Schmid CH: Summing up evidence: one answer is not always enough. Lancet 1998, 351(9096):123-127. doi:S0140-6736(97)08468-7 10.1016/50140-6736(97)08468-7.

9. Modugno F, Ness RB, Allen GO, Schildkraut JM, Davis FG, Goodman MT: Oral contraceptive use, reproductive history, and risk of epithelial ovarian cancer in women with and without endometriosis. Am J Obstet Gynecol 2004, 191(3):733-740. doi:S0002937804002819 10.1016/j. ajog.2004.03.035.

10. Jordan SJ, Green AC, Whiteman DC, Moore SP, Bain CJ, Gertig DM, Webb PM: Serous ovarian, fallopian tube and primary peritoneal cancers: a comparative epidemiological analysis. Int I Cancer 2008, 122(7):1598-1603. doi:10.1002/ijc.23287.

11. Ness RB, Dodge RC, Edwards RP, Baker JA, Moysich KB: Contraception methods, beyond oral contraceptives and tubal ligation, and risk of ovarian cancer. Ann Epidemiol, 21(3):188-196. doi:S1047-2797(10)00356-X 10.1016/j.annepidem.2010.10.002.

12. Moorman PG, Palmieri RT, Akushevich L, Berchuck A, Schildkraut JM: Ovarian cancer risk factors in African-American and white women. Am J Epidemiol 2009, 170(5):598-606. doi:kwp176 10.1093/aje/kwp176.

13. Antoniou AC, Rookus M, Andrieu N, Brohet R, Chang-Claude J, Peock S, Cook M, Evans DG, Eeles R, Nogues C, Faivre L, Gesta P, van Leeuwen FE, Ausems MG, Osorio A, Caldes T, Simard J, Lubinski J, Gerdes AM, Olah E, Furhauser C, Olsson H, Arver B, Radice P, Easton DF, Goldgar DE: Reproductive and hormonal factors, and ovarian cancer risk for BRCA1 and BRCA2 mutation carriers: results from the International BRCA1/2 Carrier Cohort Study. Cancer Epidemiol Biomarkers Prev 2009, 18(2):601-610. doi:1055-9965.EPI-08-0546 10.1158/1055-9965.EPI-08-0546.

14. Dorjgochoo T, Shu XO, Li HL, Qian HZ, Yang G, Cai H, Gao YT, Zheng W: Use of oral contraceptives, intrauterine devices and tubal sterilization and cancer risk in a large prospective study, from 1996 to 2006. Int J Cancer 2009, 124(10):2442-2449. doi:10.1002/ijc.24232.

15. Nagle CM, Olsen CM, Webb PM, Jordan SJ, Whiteman DC, Green AC: Endometrioid and clear cell ovarian cancers: a comparative analysis of risk factors. Eur J Cancer 2008, 44(16):2477-2484. doi:S0959-8049(08)00544-3 10.1016/j.ejca.2008.07.009.

16. Jordan SJ, Green AC, Whiteman DC, Webb PM: Risk factors for benign serous and mucinous epithelial ovarian tumors. Obstet Gynecol 2007, 109 (3):647-654. doi:109/3/647 10.1097/01.AOG.0000254159.75977.fa.

17. Tworoger SS, Fairfield KM, Colditz GA, Rosner BA, Hankinson SE: Association of oral contraceptive use, other contraceptive methods, and infertility with ovarian cancer risk. Am J Epidemiol 2007, 166(8):894-901. doi:kwm157 10.1093/aje/kwm157.

18. McLaughlin JR, Risch HA, Lubinski J, Moller P, Ghadirian P, Lynch H, Karlan B, Fishman D, Rosen B, Neuhausen SL, Offit K, Kauff N, Domchek S, Tung N, Friedman E, Foulkes W, Sun P, Narod SA: Reproductive risk factors for ovarian cancer in carriers of BRCA1 or BRCA2 mutations: a case-control study. Lancet Oncol 2007, 8(1):26-34. doi:S1470-2045(06)70983-4 10.1016/ S1470-2045(06)70983-4

19. Kjaer SK, Mellemkjaer L, Brinton LA, Johansen C, Gridley G, Olsen JH: Tubal sterilization and risk of ovarian, endometrial and cervical cancer. A Danish population-based follow-up study of more than 65000 sterilized women. Int J Epidemiol 2004, 33(3):596-602. doi:10.1093/ije/dyh046 dyh046.

20. McGuire V, Felberg A, Mills M, Ostrow KL, DiCioccio R, John EM, West DW, Whittemore AS: Relation of contraceptive and reproductive history to ovarian cancer risk in carriers and noncarriers of BRCA1 gene mutations. Am J Epidemiol 2004, 160(7):613-618. doi:10.1093/aje/kwh284 160/7/613.

21. Pike $M C$, Pearce $C L$, Peters $R$, Cozen W, Wan P, Wu AH: Hormonal factors and the risk of invasive ovarian cancer: a population-based case-control study. Fertil Steril 2004, 82(1):186-195. doi:10.1016/j.fertnstert.2004.03.013 S0015028204005564.

22. Risch HA, Marrett LD, Jain M, Howe GR: Differences in risk factors for epithelial ovarian cancer by histologic type. Results of a case-control study. Am J Epidemiol 1996, 144(4):363-372.

23. Rutter JL, Wacholder S, Chetrit A, Lubin F, Menczer J, Ebbers S, Tucker MA, Struewing JP, Hartge P: Gynecologic surgeries and risk of ovarian cancer in women with BRCA1 and BRCA2 Ashkenazi founder mutations: an Israeli population-based case-control study. J Natl Cancer Inst 2003, 95 (14):1072-1078.

24. Wittenberg J, Cook LS, Rossing MA, Weiss NS: Reproductive risk factors for mucinous and non-mucinous epithelial ovarian cancer. Epidemiology 1999, 10(6):761-763. 
25. Kreiger N, Sloan M, Cotterchio M, Parsons P: Surgical procedures associated with risk of ovarian cancer. Int J Epidemio/ 1997, 26(4):710-715.

26. Green A, Purdie D, Bain C, Siskind V, Russell P, Quinn M, Ward B: Tubal sterilisation, hysterectomy and decreased risk of ovarian cancer. Survey of Women's Health Study Group. Int J Cancer 1997, 71(6):948-951. doi:10.1002/ (SICI)1097-0215(19970611)71:6<948::AID-IJC6>3.0.CO;2-Y.

27. Cornelison TL, Natarajan N, Piver MS, Mettlin CJ: Tubal ligation and the risk of ovarian carcinoma. Cancer Detect Prev 1997, 21(1):1-6.

28. Miracle-McMahill HL, Calle EE, Kosinski AS, Rodriguez C, Wingo PA, Thun MJ, Heath CW Jr: Tubal ligation and fatal ovarian cancer in a large prospective cohort study. Am J Epidemiol 1997, 145(4):349-357.

29. Rosenblatt KA, Thomas DB: Reduced risk of ovarian cancer in women with a tubal ligation or hysterectomy. The World Health Organization Collaborative Study of Neoplasia and Steroid Contraceptives. Cancer Epidemiol Biomarkers Prev 1996, 5(11):933-935.

30. Nandakumar A, Anantha N, Dhar M, Ahuja V, Kumar R, Reddy S, Venugopal T, Rajanna VAT, Srinivas: A case-control investigation on cancer of the ovary in Bangalore, India. Int J Cancer 1995, 63(3):361-365.

31. Whittemore AS, Harris R, Itnyre J: Characteristics relating to ovarian cancer risk: collaborative analysis of 12 US case-control studies. II. Invasive epithelial ovarian cancers in white women. Collaborative Ovarian Cancer Group. Am J Epidemiol 1992, 136(10):1184-1203.

32. Booth M, Beral V, Smith P: Risk factors for ovarian cancer: a case-control study. Br J Cancer 1989, 60(4):592-598.

33. Shu XO, Brinton LA, Gao YT, Yuan JM: Population-based case-control study of ovarian cancer in Shanghai. Cancer Res 1989, 49(13):3670-3674.

34. Koch $\mathrm{M}$, Jenkins $\mathrm{H}$, Gaedke $\mathrm{H}$ : Risk factors of ovarian cancer of epithelial origin: a case control study. Cancer Detect Prev 1988, 13(2):131-136.

35. Koch M, Starreveld AA, Hill GB, Jenkins $H$ : The effect of tubal ligation on the incidence of epithelial cancer of the ovary. Cancer Detect Prev 1984, 7 (4):241-245

36. Mori M, Harabuchi I, Miyake H, Casagrande JT, Henderson BE, Ross RK: Reproductive, genetic, and dietary risk factors for ovarian cancer. Am J Epidemiol 1988, 128(4):771-777

37. Wu AH, Pearce CL, Tseng CC, Templeman C, Pike MC: Markers of inflammation and risk of ovarian cancer in Los Angeles County. Int J Cancer 2009, 124(6):1409-1415. doi:10.1002/ijc.24091.

38. Annegers JF, Strom H, Decker DG, Dockerty MB, O'Fallon WM: Ovarian cancer: incidence and case-control study. Cancer 1979, 43(2):723-729.

39. Luoto R, Auvinen A, Pukkala E, Hakama M: Hysterectomy and subsequent risk of cancer. Int J Epidemiol 1997, 26(3):476-483.

40. Beard CM, Hartmann LC, Atkinson EJ, O'Brien PC, Malkasian GD, Keeney GL, Melton $\sqcup$ 3rd: The epidemiology of ovarian cancer: a population-based study in Olmsted County, Minnesota, 1935-1991. Ann Epidemio/ 2000, 10 (1):14-23. doi:S1047-2797(99)00045-9.

41. Braem MG Onland-Moret NC van den Brandt PA Goldbohm RA, Peeters $\mathrm{PH}$, Kruitwagen RF, Schouten LJ: Reproductive and hormonal factors in association with ovarian cancer in the Netherlands cohort study. Am J Epidemiol, 172(10):1181-1189. doi:kwq264 10.1093/aje/kwq264.

42. Chiaffarino F, Parazzini F, Decarli A, Franceschi S, Talamini R, Montella M, La Vecchia C: Hysterectomy with or without unilateral oophorectomy and risk of ovarian cancer. Gynecol Oncol 2005, 97(2):318-322. doi:S0090-8258 (05)00085-5 10.1016/j.ygyno.2005.01.030.

43. Hankinson SE, Hunter DJ, Colditz GA, Willett WC, Stampfer MJ, Rosner B, Hennekens $\mathrm{CH}$, Speizer FE: Tubal ligation, hysterectomy, and risk of ovarian cancer. A prospective study. JAMA 1993, 270(23):2813-2818.

44. Loft A, Lidegaard O, Tabor A: Incidence of ovarian cancer after hysterectomy: a nationwide controlled follow up. Br J Obstet Gynaecol 1997, 104(11):1296-1301.

45. Parazzini F, Negri E, La Vecchia C, Luchini L, Mezzopane R: Hysterectomy, oophorectomy, and subsequent ovarian cancer risk. Obstet Gynecol 1993, 81(3):363-366.

46. Risch HA, Marrett LD, Howe GR: Parity, contraception, infertility, and the risk of epithelial ovarian cancer. Am J Epidemiol 1994, 140(7):585-597.

47. Wynder EL, Dodo H, Barber HR: Epidemiology of cancer of the ovary. Cancer 1969, 23(2):352-370.

48. Irwin $\mathrm{KL}$, Weiss NS, Lee NC, Peterson HB: Tubal sterilization, hysterectomy, and the subsequent occurrence of epithelial ovarian cancer. Am $J$ Epidemiol 1991, 134(4):362-369.

49. Tung KH, Goodman MT, Wu AH, McDuffie K, Wilkens LR, Kolonel LN, Nomura AM, Terada KY, Carney ME, Sobin LH: Reproductive factors and epithelial ovarian cancer risk by histologic type: a multiethnic case-control study. Am J Epidemiol 2003, 158(7):629-638.

50. Cramer DW, Xu H: Epidemiologic evidence for uterine growth factors in the pathogenesis of ovarian cancer. Ann Epidemiol 1995, 5(4):310-314. doi:1047-2797(94)00098-E.

51. Hakverdi AU, Taner CE, Erden AC, Satici O: Changes in ovarian function after tubal sterilization. Adv Contracept 1994, 10(1):51-56.

52. Radwanska E, Headley SK, Dmowski P: Evaluation of ovarian function after tubal sterilization. J Reprod Med 1982, 27(7):376-384.

53. Cattanach J: Oestrogen deficiency after tubal ligation. Lancet 1985, 1 (8433):847-849. doi:S0140-6736(85)92209-3.

54. Garza-Flores J, Vazquez-Estrada L, Reyes A, Valero A, Morales del Olmo A, Alba VM, Bonilla C: Assessment of luteal function after surgical tubal sterilization. Adv Contracept 1991, 7(4):371-377.

55. Wu E, Xiao B, Yan W, Li H, Wu B: Hormonal profile of the menstrual cycle in Chinese women after tubal sterilization. Contraception 1992 45(6):583-593.

56. Eliassen AH, Colditz GA, Rosner B, Hankinson SE: Tubal sterilization in relation to breast cancer risk. Int J Cancer 2006, 118(8):2026-2030. doi: 10.1002/ijc.21582.

57. Pinheiro SP, Hankinson SE, Tworoger SS, Rosner BA, McKolanis JR, Finn OJ, Cramer DW: Anti-MUC1 antibodies and ovarian cancer risk: prospective data from the Nurses' Health Studies. Cancer Epidemiol Biomarkers Prev, 19 (6):1595-1601. doi:1055-9965.EPI-10-0068 10.1158/1055-9965.EPI-10-0068.

58. Nagle CM, Bain CJ, Green AC, Webb PM: The influence of reproductive and hormonal factors on ovarian cancer survival. Int J Gynecol Cancer 2008, 18(3):407-413. doi:IJG1031 10.1111/j.1525-1438.2007.01031.x.

59. Modugno F, Ness RB, Wheeler JE: Reproductive risk factors for epithelial ovarian cancer according to histologic type and invasiveness. Ann Epidemiol 2001, 11(8):568-574. doi:S1047279701002137.

doi:10.1186/1757-2215-5-13

Cite this article as: Rice et al:: Tubal ligation, hysterectomy and ovarian cancer: A meta-analysis. Journal of Ovarian Research 2012 5:13

\section{Submit your next manuscript to BioMed Central and take full advantage of:}

- Convenient online submission

- Thorough peer review

- No space constraints or color figure charges

- Immediate publication on acceptance

- Inclusion in PubMed, CAS, Scopus and Google Scholar

- Research which is freely available for redistribution

Submit your manuscript at www.biomedcentral.com/submit
C BioMed Central 